Methods: Total of 39766 patients were analysed for anti-Sm antibody using the EURO lineimmuno assay skit, of which 1208 cases are positive for anti-Sm. All cases with positive anti-Sm were divided into true anti-Sm group and implausible group according to the result of Sm/RNP band. The proportion of clinical diagnosis including autoimmune diseases (AIDs) and non-immune diseases (NAIDs) and the level of some clinical serum indexeswere retrospectively analysed for the implausible anti-Sm cases

Results: Implausible anti-Sm were evidenced in 271/1208 (22.42) of all positive anti-Sm cases. Among patients with positive anti-Sm, 177 cases have definitive diagnoses $(65.31 \%, 177 / 271)$, with autoimmune diseases accounting for $68.36 \%$ (121/177), of whom 96 had systemic lupus erythematosus (SLE) (79.34\%). In addition, there are 5 implausible cases with rheumatoid arthritis (RA), 3 with Sjogren syndrome (SS) and 10 with mixed connective tissue diseases (MCTDs). The titer of antinuclear antibodies (ANA) in patients with implausible anti-Sm is lower than ones with true anti-Sm. Impausible group have the same gender ratio, mean onset age and frequency of autoantibodies with true group. The prevalence of one of NAIDs, kidney disease, was significantly lower in implausible group than in true group ( $\mathrm{X} 2=3.841, \mathrm{p}=0.05)$.

Conclusions: Implausible anti-Sm have great diagnostic value in AIDs just as true anti-Sm. Patients with implausible anti-Sm have less incidence of evolving to autoimmune injury and kidney injury. Patients with implausible anti-Sm but without autoimmune diseases may be potential autoimmune disease victims.

Acknowledgements: This work was supported by fund of Department of Science and Technology of Sichuan Province, Grant No. 2016 JY0035 and the fund of Health and Family Planning Commission of Sichuan Province, Great. No.16pg326.

Disclosure of Interest: None declared

DOI: 10.1136/annrheumdis-2018-eular.2368

\section{AB0641 CORRELATION BETWEEN PHYSICIANS AND PATIENTS IN THE ASSESSMENT OF DISEASE ACTIVITY IN SYSTEMIC LUPUS ERYTHEMATOSUS}

Y. Nasa $^{1,2}$, N. Yokokawa ${ }^{2}$, H. Takahashi ${ }^{2}$, E. Takamasu ${ }^{2}$, S. Sugii ${ }^{2} .{ }^{1}$ First Department of Internal Medicine, Kansai Medical University, Osaka; ${ }^{2}$ Department of Rheumatic Diseases, Tokyo Tama Medical Metropolitan Hospital, Tokyo, Japan

Background: In the assessment of systemic lupus erythematosus (SLE), laboratory data and clinical findings have been more focused than patient-reported outcomes which reflect quality of Life. Previous studies showed discrepancy between patients and physicians in assessments of disease activity in SLE. ${ }^{1}$ The Systemic Lupus Activity Questionnaire (SLAQ), a lupus disease activity index which consisted of only patient reported outcomes, and SLE Disease Activity Index 2000 (SLEDAI-2K) were poorly corelated. ${ }^{2}$

Objectives: We aimed to investigate the correlation between SLAQ and SLEDAI2K scores in those who had discordance between patients' VAS and physicians' VAS or not.

Methods: Both SLEDAI-2K and SLAQ were routinely measured at every outpatient visit in all SLE patients. We analysed the data between 9/12/2017 and 12/31/ 2017. Patients were divided into concordance(|patients' VAS-physicians' VAS $<25$ ) or discordance group (|patients' VAS-physicains' VAS $\geq 25)$. ${ }^{3}$ We measured correlation between SLAQ score vs SLEDAI-2K or SLEDAI-2K-nolab scores by Spearman's correlation in the concordance group and the discordance group. Comparison between the concordance group and the discordance group were perfomed using chi-squared test for categorical variables and Student t-test Welch's t-test or Mann-Whitney U test for continuous variables.

Results: Total 130 patients were analysed; $91 \%$ of female, a mean age (SD) of $44^{15}$ years, steroid use of $91 \%$, immunosuppressant use of $54 \%$, HCQ use of $70 \%$. Median SLAQ, SLEDAI-2K and SLEDAI-2K-nolab scores were 4 [IQR: 27], 4 [IQR: 2-4] and 0 [IQR: 0-2], respectively. Among them, 86 (66\%) were classified in the concordance group. The SLAQ scores were weakly correlated with the SLEDAI-2K scores $(\rho=0.228, \mathrm{p}=0.009)$, and with SLEDAI-2K-nolab scores $(\rho=0.352, \mathbf{p}<0.001)$. In the concordance group, the SLAQ scores correlated with SLEDAI-2K scores $(\rho=0.327, \mathbf{p}=0.002)$ and SLEDAI-2K-nolab scores $(\rho=0.523$, $\mathrm{p}<0.001)$. The pain VAS and RAPID3 in the discordance group were significantly higher than those in the concordance group (30.77 \pm 26.54 vs $10.71 \pm 14.31$, $\mathrm{p}<0.001,8.11 \pm 5.87$ vs $2.97 \pm 3.23, \mathrm{p}<0.001$, repectively). In the discordance group, the SLAQ scores were not correlated with SLEDAI-2K scores $(\rho=-0.029$, $\mathrm{p}=0.849)$ and with SLEDAI-2K-nolab scores $(\rho=-0.083, \mathrm{p}=0.957)$.
Abstract AB0641 - Table 1. LLDAS: Lupus low Disease Activity State *Student t-test **Welch's t-test $₫$ Mann-Whitney U test

\begin{tabular}{|c|c|c|c|}
\hline & concordance Eroup $(n=86)$ & discordance group $(n=44)$ & $p$ value \\
\hline Age (mean, SD) & $41.74(14.74)$ & $49.00(15.63)$ & $0.010 *$ \\
\hline Sex (female) (\$) & $78(90.7)$ & $40(90.9)$ & 1.000 \\
\hline Disease duration (year. mean, SD) & $10.78(9.47)$ & $12.18(10.75)$ & $0.447 *$ \\
\hline Antimalarial (\&) & $61(70.9)$ & $30(68.2)$ & 0.903 \\
\hline Immunosuppresant (\$) & $47(54.7)$ & $23(52.3)$ & 0.943 \\
\hline PSLdoses (mean, SD) (mg) & $5.13(4.48)$ & $5.59(5.95)$ & 0.6291 \\
\hline LLDAS (\$) & $48(55.8)$ & $22(50.0)$ & 0.658 \\
\hline MDHAQ (mean, SD) & $0.20(0.55)$ & $0.33(0.46)$ & $0.015 \uparrow$ \\
\hline Pain (100mm VAS) (mean, SD) & $10.71(14.31)$ & $30.77(26.54)$ & $<0.001 *$ \\
\hline Patietnt global (100mm VAS) (mean, SD) & $14.43(15.72)$ & $39.57(23.25)$ & $<0.001 * *$ \\
\hline Physician global assessment (100mm VAS) (m & $15.64(13.64)$ & $15.07(15.39)$ & $0.829 *$ \\
\hline RAPID3 (mean, SD) & $2.97(3.23)$ & $8.11(5.87)$ & $<0.001 * *$ \\
\hline SLAQ score (mean, SD) & $4.63(3.93)$ & $6.16(4.89)$ & $0.056 *$ \\
\hline SLEDAl-2K (mean, SD) & $3.50(3.14)$ & $3.82(2.90)$ & $0.575 *$ \\
\hline SLEDAI-2K-nolab (mean. SD) & $0.92(2.22)$ & $1.73(2.68)$ & 0.0301 \\
\hline
\end{tabular}

Conclusions: The correlation between SLAQ vs SLEDAI-2K or SLEDAI-2K nolab scores was exhibited in the concordance group. Musculoskeletal pain may be associated with the discordance between patients' and physicians' assessment.

\section{REFERENCES}

[1] Karlson EW, et al. Vlidation of a Systemic Lupus Activity Questionnaire (SLAQ) for population studies. Lupus 2003;12:280-286.

[2] Okamoto $\mathrm{Y}$, et al. Validation of the Japanese version of the Systemic Lupus Activity Questionnaire that includes physician-based assessments in a large observational cohort. Lupus 2016;25:486-495.

[3] Barton JL, et al. Patient-physician discordance in assessments of a global disease severity in rheumatoid arthritis. Arthritis Care Res 2010;62:85764

Disclosure of Interest: None declared

DOI: 10.1136/annrheumdis-2018-eular.2743

\section{AB0642 GLUCOCORTICOID INTAKE, HYPERGLYCEMIA AND OSTEOPOROSIS IN PATIENTS WITH AUTOIMMUNE DISEASES}

Y. Jiang, G. Du, X. Wu, Y. Xie, M. Zhao, Z. Liao, J. Gu. Rheumatology, The Third Affiliated Hospital of Sun Yat-Sen University, Guangzhou, China

Background: Autoimmune diseases (AID) are often treated with glucocorticoids Glucocorticoids have a number of substantial side effects in human body, including hyperglycemia and osteoporosis.

Objectives: Our study was to investigate the use of glucocorticoid in patients with autoimmune diseases and its negative influence on blood glucose and osteoporosis in the patients who receiving glucocorticoid treatment.

Methods: Patients with autoimmune diseases were enrolled from July to Decem ber in 2017 in rheumatology department of the Third Affiliated Hospital of Sun Yatsen University. Demographic information, family history, past medical history, and clinical information were collected by two rheumatologists, including years of having glucocorticoids for treatment, largest dose of methylprednisolone, current dose of glucocorticoids. Blood glucose, glycosylated haemoglobin, and bone min eral density was required. The Statistical Package for Social Sciences (SPSS) software version 21 was used for all data management and analysis.

Results: Of all the 75 patients, $15(20 \%)$ were male patients. $14.7 \%$ had primary education, while $26.6 \%$ received education in university. Numbers of the patients were stated as follows. Lupus, 29; rheumatoid arthritis, 4; Sjogren's syndrome 10; systemic sclerosis, 10; myositis, 4; mixed connective tissue disease, 1 ; autoimmune hepatitis, 1 ; vasculitis, 5; other diseases, 11 . Mean age was $40.29 \pm 14.64$ years. Mean disease duration was $4.74 \pm 6.62$ years. $3(4 \%)$ patients had family history of diabetes. $3(4 \%)$ patients had past medical history of diabetes. Mean duration of taking glucocorticoids was $3.30 \pm 4.40$ years. $13(17.3 \%)$ of the patients underwent high dose of glucocorticoid intravenous pulse $(120 \mathrm{mg}$ to $1000 \mathrm{mg}$ of methylprednisolone). Current dose of glucocorticoids was $5.30 \pm 3.96$ tablets of methylprednisolone. Mean blood glucose was $4.61 \pm 0.92 \mathrm{mmol} / \mathrm{L}$. Mean glycosylated haemoglobin was $5.46 \pm 0.84$. 2 patients were found to have diabetes in this study. 5 other patients were found to have higher blood sugar than normal range (3.9-6.1 mmol/L, according to our laboratory). 14 (18.7\%) of the patients had osteoporosis according to BMD scores. In 17 patients who had receiving glucocorticoids for more than five years, $3(17.6 \%)$ patients were found to have higher 